\title{
Validation of the Sinhala version of the Montreal Cognitive Assessment in screening for dementia
}

\author{
S Karunaratne ${ }^{1,}$ R Hanwella ${ }^{2}$, V de Silva ${ }^{2}$ \\ (Index words: Montreal Cognitive Assessment, dementia, validation, Sinhala, scales)
}

\begin{abstract}
Objectives To validate the Sinhala version of the Montreal Cognitive Assessment (MoCA) scale in screening for dementia.

Methods The MoCA translation and cultural adaptation was carried using a combined qualitative and quantitative approach. Sample size was calculated to detect a targeted sensitivity of $85 \%$ and a specificity of $85 \%$. Sample consisted of 49 participants diagnosed with dementia of the Alzheimer's type (AD) according to DSM-IV criteria and 49 normal controls (NC) aged $\geq 50$ years. All subjects were administered the Mini Mental State Examination (MMSE) and MoCA Sinhala version (MoCA-S). Concurrent validity was assessed using Pearson correlation coefficients between the MoCA-S scores and MMSE scores. Criterion validity was assessed using receiver operating characteristic (ROC) analysis.
\end{abstract}

Results Mean MoCA scores between NC (26.71, SD 2.4) and $A D$ group (16.78, SD 5.9) were significantly different $(t=10.8, p<0.001)$. Cronbach's alpha of 0.818 indicated good internal consistency. Attention (digit span, sustained attention, and the serial 7 calculation task) had the highest discriminant ability followed by visuospatial skills (trail making, cube drawing and clock drawing). Naming had poor discriminant ability. There was a high, positive correlation between MoCA-S total scores and MMSE total scores. $(r=0.907, p<0.001)$. The area under the ROC curve was $0.975(95 \% \mathrm{Cl} 0.94-1.0)$ for the MoCA and 0.928 (95\% Cl 0.87-0.98) for MMSE. A cut-off value of 24 provided the best balance between sensitivity (98.0 7\%) and specificity (79.6\%).

Conclusion MoCA-S is a valid and reliable instrument which can be used as a brief screening instrument for dementia in Sri Lanka.

Ceylon Medical Journal 2011; 56: 147-153

\section{Introduction}

With the increase in the elderly population in Sri Lanka, dementia has become an important health problem.
A survey of a semi-urban population in Sri Lanka found an overall prevalence of dementia of 3.98\% (95\% CI 2.6-5.7) among those aged $\geq 65$ years [1]. Many with dementia remain undiagnosed for years. Early diagnosis is important because intervention with cognitive stimulation, physical exercise, aggressive treatment of risk factors and pharmacological interventions can slow down cognitive deterioration [2].

Apart from lack of public knowledge about dementia, lack of awareness by the medical profession regarding diagnostic criteria and cognitive testing contributes to low rates of detection. In Sri Lanka in a general medical ward $55.2 \%$ of patients over 65 years who had a secondary education scored at or below 23 on the MMSE [3]. Many of these patients would not have been identified by clinicians as requiring interventions for cognitive impairment.

Clinical diagnosis of dementia is made using standardised operational criteria such as the DSM-IV criteria (Diagnostic and Statistical Manual) or the NINCDSADRDA criteria (National Institute of Neurological and Communicative Disorders and Stroke and Alzheimer's Disease and Related Disorders Association) [4, 5]. Neuropsychological tests are used in screening for dementia and are particularly useful for identifying those with early stages of the disorder. Testing also helps detail the areas of cognitive impairment, assess the severity and monitor the progress of the disease.

New knowledge about Alzheimer's and other dementias has led to the recognition of intermediate clinical states where cognitive impairment does not reach the threshold for dementia [6]. Therefore sensitive screening tools which can identify early stages of the disease are being developed.

Mild cognitive impairment (MCI) is defined as an intermediate clinical state between normal aging and dementia and is thought to precede and progress to dementia in many cases [7]. There are several definitions of MCI. Diagnosis of MCI according to Peterson criteria

${ }^{1}$ University Psychiatry Unit, National Hospital of Sri Lanka, Colombo, ${ }^{2}$ Department of Psychological Medicine, Faculty of Medicine, Colombo, Sri Lanka.

Correspondence: VdeS, e-mail: <varunidesilva2@yahoo.co.uk>. Received 5 July and revised version accepted 16 September 2011. Competing interests: none declared. 
require subjective complaints of gradual memory loss over 6 months reported by the patient or the family, objective evidence of memory loss, general preservations of other cognitive domains and preserved functioning in terms of activities of daily living [7].

Complex test batteries such as the CAMDEX (Cambridge Mental Disorders of the Elderly Examination) or the CAMCOG which forms part of the CAMDEX are too detailed to be used as bed side tests or as screening tools [8]. Instead, brief cognitive assessment tools such as the Mini Mental State Examination (MMSE) are widely used in clinical practice. The MMSE has been translated and culturally adapted for use in Sri Lanka [9]. However, it has several disadvantages. The MMSE shows high sensitivity for moderate-to-severe cognitive impairment but lower sensitivity for mild degrees of cognitive impairment [10-12]. The MMSE also has very low sensitivity in detecting mild cognitive impairment (MCI) [13].

The MoCA (Montreal Cognitive Assessment) was developed as a tool to screen patients with mild cognitive complaints who usually perform in the normal range on the MMSE. It also addresses the difficulties of detecting early dementia as many of these patients score over 26 in the MMSE. It is able to differentiate between normal and MCI as well as between MCI and early dementia. The original validation study of the MoCA reported a sensitivity of $100 \%$ and specificity of $87 \%$ in detecting mild Alzheimer's disease (cut-off 26). It reported a sensitivity of $90 \%$ in detecting MCI [14].

MoCA has been translated into 36 languages [15]. Its validity in detecting cognitive impairment in different clinical populations with Parkinson disease, brain metastases and strokes has been established [16-18]. The high sensitivity of the MoCA in detecting MCI and dementia has been confirmed by studies carried out in many countries [19-23].

The MoCA covers eight cognitive domains; visuospatial skills, executive functions, short term memory recall, attention, concentration, working memory, language and orientation. It gives a maximum score of 30.

Only a few neuropsychological tests have been translated and validated for use in Sri Lanka. Because the MoCA is a sensitive tool for detecting early dementia which can easily be administered as a bedside test, and is available in the public domain, we decided to translate and culturally adapt the MoCA and investigate its validity in detecting dementia in a Sri Lankan population.

\section{Methods}

The translation of the MoCA and cultural adaptation was carried using a combined qualitative and quantitative approach [24]. Translation of the instrument alone was not adequate and adaptation appropriate to the local culture was necessary for certain items. Details of changes done in the process of adaptation are given in Table 1.

The translated scale was back translated into English by a bilingual expert who was not familiar with the original MoCA scale and then compared with the original scale. The translated instrument was pre-tested on a group of 20 individuals from the community.

Sample size was calculated to detect a targeted sensitivity of $85 \%$ and a specificity of $85 \%$ [25]. The study was conducted in Colombo. The study sample consisted of 49 participants diagnosed as having dementia of the Alzheimer's type (AD) according to DSM-IV criteria and 49 normal controls (NC) aged $\geq 50$ years who were recruited from the community. In both groups those with visual and hearing impairment, those who were illiterate and people with mental retardation were excluded as these deficits compromise the ability to complete the test. Normal controls underwent a screening clinical assessment to exclude the presence of dementia. In this group people with complaints of memory or cognitive impairment, history of head trauma, stroke or depressive illness were excluded.

Patients with dementia were recruited from the University Psychiatry Unit of the National Hospital of Sri Lanka, Colombo. Dementia of the Alzheimer's type (AD) was diagnosed according to DSM-IV criteria after clinical interview by a consultant psychiatrist. All patients with dementia had biochemical screening and a neuro-imaging tests (CT scan or MRI) to exclude other causes of dementia and cognitive impairment.

All participants were administered the MMSE and MoCA Sinhala version (MoCA-S) by a Senior Registrar or Registrar in Psychiatry trained in the administration of the MoCA and the MMSE. To correct for education effects, one point was added to the total MoCA-S score for participants with $\leq 12$ years of education (if total MoCA score was $<30$ ) as recommended by authors of the scale.

Internal consistency was measured using Cronbach's alpha. Concurrent validity was assessed using Pearson correlation coefficients between the MoCA-S scores and MMSE scores. Criterion validity was assessed using receiver operating characteristic (ROC) analysis which gave the sensitivity and specificity of the MoCA at different cut-off points. Statistical analysis was carried out using Stata.

Written informed consent was obtained from all participants. Ethical approval was obtained from the Ethics Review Committee of the National Hospital of Sri Lanka. 
Table 1. Changes in the process of translation and adaptation of the MoCA

\begin{tabular}{|c|c|c|c|}
\hline & $\begin{array}{l}\text { MoCA original English } \\
\text { version }\end{array}$ & $\begin{array}{l}\text { MoCA Sinhala } \\
\text { version }\end{array}$ & Reason for change \\
\hline Trail making test & $\begin{array}{l}\text { First five letters of the } \\
\text { English alphabet }\end{array}$ & $\begin{array}{l}\text { Replaced by the first five } \\
\text { letters of the Sinhala alphabet }\end{array}$ & \\
\hline Naming & $\begin{array}{l}\text { Pictures of lion, } \\
\text { rhinoceros, camel }\end{array}$ & $\begin{array}{l}\text { Pictures of lion, } \\
\text { rabbit and elephant }\end{array}$ & $\begin{array}{l}\text { Pilot study found normal } \\
\text { healthy people had } \\
\text { difficulty identifying } \\
\text { rhinoceros and camel }\end{array}$ \\
\hline Memory & $\begin{array}{l}\text { Face, velvet, church, } \\
\text { daisy, red }\end{array}$ & $\begin{array}{l}\text { Velvet replaced by Sinhala } \\
\text { word for cotton, church replaced } \\
\text { by Sinhala word for temple, daisy } \\
\text { replaced by a flower 'Araliya' }\end{array}$ & $\begin{array}{l}\text { People unfamiliar with } \\
\text { velvet. Majority of Sinhala } \\
\text { speaking people are } \\
\text { Buddhist and are more } \\
\text { familiar with the term } \\
\text { temple. Daisy replaced by } \\
\text { name of a common flower } \\
\text { in Sri Lanka }\end{array}$ \\
\hline List of letters & $\begin{array}{l}\text { List of letters of the English } \\
\text { alphabet }\end{array}$ & $\begin{array}{l}\text { Replaced by Sinhala letter with a } \\
\text { corresponding sound }\end{array}$ & \\
\hline $\begin{array}{l}\text { Language } \\
\text { Sentence } 1\end{array}$ & $\begin{array}{l}\text { "I know that John is the one } \\
\text { to help today". }\end{array}$ & $\begin{array}{l}\text { Sentence translated to retain same } \\
\text { meaning and same number of words. } \\
\text { Name “John” replaced with "Nimal”. }\end{array}$ & $\begin{array}{l}\text { Use of a common } \\
\text { name in Sri Lanka. }\end{array}$ \\
\hline $\begin{array}{l}\text { Language } \\
\text { Sentence } 2\end{array}$ & $\begin{array}{l}\text { "The cat always hid under } \\
\text { the couch when dogs were } \\
\text { in the room". }\end{array}$ & $\begin{array}{l}\text { Sentence translated to retain } \\
\text { same number of words. The meaning } \\
\text { has changed slightly. Back translation } \\
\text { of Sinhala sentence is as follows } \\
\text { "When the dog barks the cat in our } \\
\text { house runs and hides under the table". }\end{array}$ & $\begin{array}{l}\text { A meaningful sentence } \\
\text { with same number of } \\
\text { words and similar } \\
\text { meaning. }\end{array}$ \\
\hline Verbal fluency & $\begin{array}{l}\text { Name maximum number of } \\
\text { words beginning with } \\
\text { letter "F" }\end{array}$ & $\begin{array}{l}\text { Replaced with Sinhala } \\
\text { letter with sound "S" }\end{array}$ & $\begin{array}{l}\text { The corresponding Sinhala } \\
\text { "F" is not used commonly } \\
\text { and there are very few Sinhala } \\
\text { words starting with that letter. } \\
\text { Therefore it was replaced } \\
\text { with another letter. }\end{array}$ \\
\hline
\end{tabular}

\section{Results}

The mean age of the sample was 64.12 years (SD 12.04) and mean years of education was 10.34 (SD 3.09). Females consisted $59.2 \%$ of the sample. The demographic characteristics of the AD group and the NC are shown in Table 2. There was no significant difference between the two groups in gender distribution $\left(\chi^{2}=0.169, \mathrm{df}=1, p=0.68\right)$. The AD group was significantly older than the NC $(\mathrm{t}=6.1$, $p<0.001)$. Normal controls had more years of education than AD group ( $\mathrm{t}=2.57, p=0.012)$.

There were significant differences in mean MoCA scores between NC (26.71, SD 2.4) and AD group (16.78, SD 5.9) $(t=10.8, p<0.001)$. This difference remained significant after adjusting for age and years of education ( $\mathrm{F}=54.84, p<0.001)$. Mean MMSE score between normal controls (29.11, SD 1.14) and dementia participants (20.38, SD 6.2) were also significantly different ( $\mathrm{t}=-9.48, p<0.001)$. There was no significant difference in mean MoCA scores between males and females ( $\mathrm{t}=0.857, p=0.393)$. Age and MoCA scores were negatively correlated $(r=-.506, p<0.001)$ and years of education and MoCA scores were positively correlated $(\mathrm{r}=.344, p<0.001)$. 
Table 2. Demographic characteristics of participants

\begin{tabular}{lll}
\hline & $\begin{array}{l}\text { Alzheimer's } \\
\text { dementia }\end{array}$ & $\begin{array}{l}\text { Normal } \\
\text { controls }\end{array}$ \\
\hline $\begin{array}{l}\text { Age years mean } \\
\text { (standard } \\
\text { deviation) }\end{array}$ & 70.45 (SD 10.19) & 57.8(SD 10.35) \\
$\begin{array}{l}\text { Female N(\%) } \\
\begin{array}{l}\text { Education } \\
\text { years (SD) }\end{array}\end{array}$ & 9.54(3.1) & $16(60.0)$ \\
$\begin{array}{l}\text { MMSE mean } \\
\text { scores (SD) }\end{array}$ & $20.38(6.2)$ & $29.1(1.15)$ \\
\hline
\end{tabular}

\section{Reliability}

Cronbach's alpha value of 0.818 indicated good internal consistency. It was comparable with the internal consistency of MMSE in this sample of 0.783.

Table 3 shows the inter-item correlation. There was high correlation between domains of visuospatial skills and attention. Naming had low correlation with all other domains. Low correlation between abstraction and visuospatial skills, and abstraction and orientation indicated that these items tested different constructs.
Table 4 shows the item-test and item-rest correlation which indicate the discriminant ability of an item. Itemtest and item-rest correlations show that attention (digit span, sustained attention, and the serial 7 calculation task) had the highest discriminant ability followed by visuospatial skills (trail making, cube drawing and clock drawing). Naming had poor correlation with total scores indicating poor discriminant ability.

Cronbach's Alpha when a particular item is removed is a good measure of that item's contribution to the entire test's performance. Cronbach's alpha increased when the item 'naming' was removed indicating that the contribution of this item to the scale was poor.

There was a high, positive correlation between MoCA total scores and MMSE total scores. ( $\mathrm{r}=0.907, p<0.001$ ). The capacity of the MoCA and the MMSE to differentiate Alzheimer's dementia from normal controls was assessed using receiver-operating characteristic (ROC) methods (Figure 1). Clinical diagnosis made according the DSM-IV criteria was used as the gold standard. The area under the ROC curve was 0.975 (95\%CI 0.94-1.0) for the MoCA and 0.928 (95\%CI 0.87-0.98) for MMSE. The difference was not statistically significant $(p=0.069)$.

Table 5 presents the sensitivity and specificity of the MoCA-S at different cut-off values. At a cut-off value of 24 the sensitivity was $98.07 \%$ and specificity $79.6 \%$ and provided the best balance between sensitivity and specificity. For the MMSE at a cut-off value of 26, sensitivity was $80 \%$ and specificity $100 \%$.

Table 3. Inter-item correlation matrix

\begin{tabular}{lccccccc}
\hline & Visuospatial & Naming & Attention & Language & Abstraction & $\begin{array}{c}\text { Delayed } \\
\text { recall }\end{array}$ & Orientation \\
\hline Visuospatial & 1.00 & .389 & .700 & 505 & .392 & .418 & .642 \\
Naming & .389 & 1.0 & .359 & .236 & .291 & .056 & .246 \\
Attention & .700 & .359 & 1.0 & .570 & .448 & .486 & .560 \\
Language & .505 & .236 & .570 & 1.0 & .342 & .466 & .443 \\
Abstraction & .392 & .291 & .448 & .342 & 1.0 & .236 & .374 \\
Delayed recall & .418 & .056 & .486 & .466 & .236 & 1.0 & .473 \\
Orientation & .642 & .246 & .561 & .443 & .374 & .473 & 1.0 \\
\hline
\end{tabular}

Table 4. Item-test and item-rest correlation

\begin{tabular}{lccc}
\hline & Item-test correlation & Item-rest correlation & Cronbach's alpha if item removed \\
\hline Visuospatial & 0.831 & 0.736 & .76 \\
Naming & 0.387 & 0.334 & .83 \\
Attention & 0.844 & 0.748 & .76 \\
Language & 0.705 & 0.614 & .79 \\
Abstraction & 0.541 & 0.460 & .82 \\
Delayed recall & 0.711 & 0.536 & .80 \\
Orientation & 0.813 & 0.671 & .78 \\
\hline
\end{tabular}




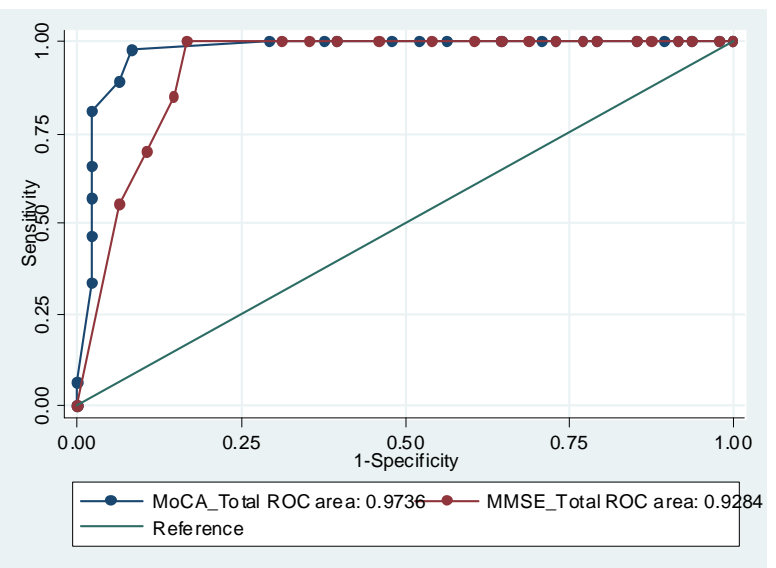

Figure 1. ROC curve analysis of the MoCA-Sinhala and the Mini-Mental State Examination (MMSE) to detect dementia.

\section{Table 5. Discriminant validity of the Montreal Cognitive Assessment (MoCA) for diagnosis of dementia}

\begin{tabular}{ccc}
\hline Cut off & Sensitivity & Specificity \\
\hline 22 & .918 & 95.9 \\
23 & .939 & 87.8 \\
24 & .980 & 79.6 \\
25 & .980 & 65.3 \\
26 & .980 & 57.1 \\
27 & .980 & 46.9 \\
\hline
\end{tabular}

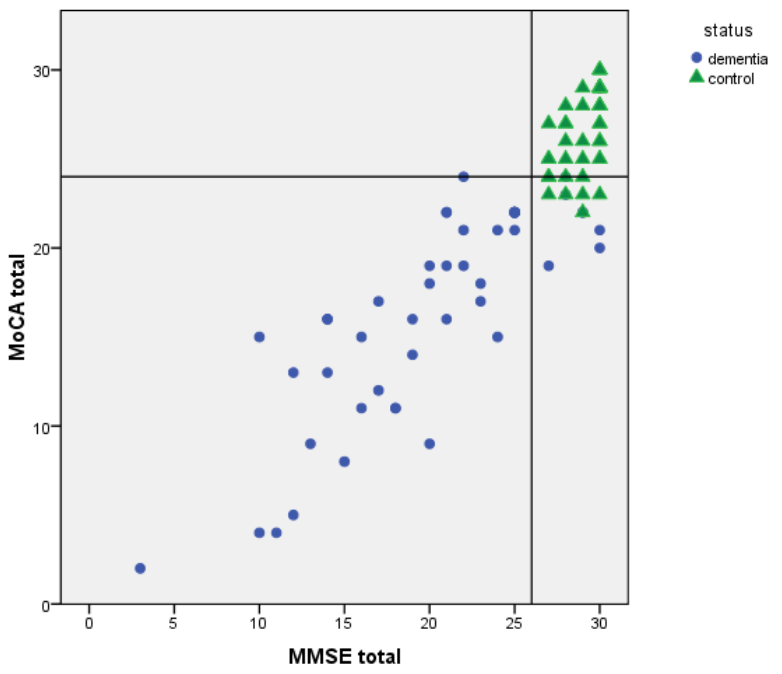

Figure 2. MoCA and MMSE scores for normal controls (NCs) and subjects with Alzheimer's disease (AD).
A scatter plot of MMSE and MoCA scores is shown in Figure 2. All dementia patients scored in the abnormal range of the MoCA but three dementia patients scored in the normal range of the MMSE (cut off value 26). All normal controls scored in the normal range of the MMSE but six healthy controls scored in the abnormal range of the MoCA (cut off value 26).

\section{Discussion}

This paper describes the psychometric properties of the MoCA a brief cognitive assessment tool which was translated to Sinhala and adapted for use in a Sri Lankan population. The translated MoCA demonstrated good internal consistency and concurrent validity as demonstrated by high correlation with MMSE scores. At a cut off score of 24 the MoCA-S had a sensitivity of $98 \%$ and specificity of $79.6 \%$ in detecting dementia.

The ideal cognitive screening instrument should be brief enough for use in clinical practice, have the ability to differentiate early dementia and MCI from normal aging, be sensitive to changes in patients' cognitive abilities over time, and should not be influenced by educational attainment and age. The MoCA has many of these properties.

The original validation study of the MoCA recommended a cut off score of 26 . We found that the specificity at a cut off of 26 was lower than at 24 (57.1\% vs.79.6\%) although the sensitivity was the same. Other studies too have found better sensitivity and specificity at lower scores of 22/23 [20, 26, 27]. It has been suggested that using the higher cut off of 26 could lead to misclassification of some normal individuals as cognitively impaired [27].

Item analysis in the original study found that the domains of visuospatial skills (trail making, cube drawing and clock drawing), attention (digit span, sustained attention, and the serial 7 calculation task), abstraction, and orientation had high discriminant ability. We too found the domains of visuospatial skills and attention to have high discriminant ability.

Naming had poor ability to discriminate between AD and normal controls. Results indicate that deleting this item would improve internal consistency of the scale. This was one item that was adapted by replacing the pictures of rhinoceros and camel with rabbit and lion. Since the original validation study did not report poor performance of this item it is important to consider replacing the items of rabbit and elephant in the Sinhala version with less familiar animals.

The value of MoCA as a screening tool in detecting MCI and early dementia has already been established. MoCA has some advantages over the MMSE especially in people with early cognitive impairment. The MoCA had excellent sensitivity (98\%) at a cut off value of 24 while the MMSE had excellent specificity (100\%) at a cut off of 
26. In our study we found that three patients with dementia scored within the normal range of the MMSE. All three of these patients scored below the MoCA cut off. All dementia patients scored in the abnormal range of the MoCA. Therefore our study confirms the finding that persons who score in the normal range of the MoCA are extremely unlikely to have AD [14].

There were several limitations in our study. Because prevalence of dementia is low a "case-control” sampling design was chosen instead of screening a large population. Potential bias of using this sampling design is that the range of illness severity may not be included. This was overcome to some extent by selecting the sample from patients presenting for out-patient assessment which enabled us to include a wider spectrum of illness severity. Some items of the MoCA cannot be completed by those who are illiterate. Therefore we cannot recommend using the MoCA in illiterate people. We also did not include a group with mild cognitive impairment. Therefore we were unable to assess the ability of the MoCA to differentiate normal from MCI and MCI from AD.

The findings from our study suggest that the MoCA$\mathrm{S}$ can be used as a brief screening instrument in Sri Lanka. Because of the high sensitivity of the MoCA in detecting cognitive impairment those scoring in the abnormal range could be assessed in more detail for MCI and dementia. The MoCA Sinhala version could be accessed at www.mocatest.org

\section{Acknowledgement}

We wish to thank Z. Nasreddine MD for granting us permission to translate the MoCA.

\section{References}

1. de Silva HA, Gunatilake SB, Smith AD. Prevalence of dementia in a semi-urban population in Sri Lanka: report from a regional survey. International Journal of Geriatric Psychiatry 2003; 18: 711-5.

2. Emery VO. Alzheimer disease: are we intervening too late? Journal of Neural Transmission 2011; 118:1361-78.

3. Rodrigo C, Perera S, Adhikari M, et al. Cognitive impairment and symptoms of depression among geriatric patients in a tertiary care unit in Sri Lanka. Indian Journal of Psychiatry 2010; 52: 279-80.

4. American Psychiatric Association: Diagnostic and Statistical Manual of Mental Disorders, Fourth Edition, Washington D.S.: American Psychiatric Association; 2000.

5. Dubois B, Feldman HH, Jacova C, et al. Research criteria for the diagnosis of Alzheimer's disease: revising the NINCDS-ADRDA criteria. Lancet Neurology 2007; 6: 734-46.

6. Kryscio RJ, Schmitt FA, Salazar JC, et al. Risk factors for transitions from normal to mild cognitive impairment and dementia. Neurology 2006; 66: 828-32.
7. Petersen RC, Doody R, Kurz A, et al. Current concepts in mild cognitive impairment. Archives of Neurology 2001; 58: 1985-92.

8. Huppert FA, Brayne C, Gill C, et al. CAMCOG - a concise neuropsychological test to assist dementia diagnosis: sociodemographic determinants in an elderly population sample. British Journal of Clinical Psychology 1995; 34: 529-41.

9. de Silva HA, Gunatilake SB. Mini mental state examination in Sinhalese: a sensitive test to screen for dementia in Sri Lanka. International Journal of Geriatric Psychiatry 2002; 17: $134-9$.

10. Tombaugh TN, McIntyre NJ. The mini mental state examination: a comprehensive review. Journal of the American Geriatrics Society 1992; 40: 922-35.

11. Wind AW, Schellevis FG, Van Staveren G, et al. Limitations of the mini mental state examination in diagnosing dementia in general practice. International Journal of Geriatric Psychiatry 1997; 12: 101-8.

12. Tangalos EG, Smith GE, Ivnik RJ, et al. The mini mental state examination in general medical practice: clinical utility and acceptance. Mayo Clinic Proceedings 1996; 71: 829-37.

13. Tang-Wai DF, Knopman DS, Geda YE, et al. Comparison of the short test of mental status and the mini-mental state examination in mild cognitive impairment. Archives of Neurology 2003; 60: 1777-81.

14. Nasreddine ZS, Phillips NA, Bedirian V, et al. The Montreal Cognitive Assessment, MoCA: a brief screening tool for mild cognitive impairment. Journal of the American Geriatrics Society 2005; 53: 695-9.

15. Nasreddine DZ. The Montreal Cognitive Assessment. Available from: http://www.mocatest.org/.

16. Olson RA, Chhanabhai T, McKenzie M. Feasibility study of the Montreal Cognitive Assessment (MoCA) in patients with brain metastases. Support Care in Cancer 2008; 16: 1273-8.

17. Godefroy O, Fickl A, Roussel M, et al. Is the Montreal Cognitive Assessment superior to the mini-mental state examination to detect poststroke cognitive impairment?: a study with neuropsychological evaluation. Stroke 2011; 42: 1712-6.

18. Hoops S, Nazem S, Siderowf AD, et al. Validity of the MoCA and MMSE in the detection of MCI and dementia in Parkinson disease. Neurology 2009; 73: 1738-45.

19. Duro D, Simoes MR, Ponciano E, Santana I. Validation studies of the Portuguese experimental version of the Montreal Cognitive Assessment (MoCA): confirmatory factor analysis. Journal of Neurology 2010; 257: 728-34.

20. Lee JY, Dong Woo L, Cho SJ, et al. Brief screening for mild cognitive impairment in elderly outpatient clinic: validation of the Korean version of the Montreal Cognitive Assessment. Journal of Geriatric Psychiatry and Neurology 2008; 21: 104-10.

21. Smith T, Gildeh N, Holmes C. The Montreal Cognitive Assessment: validity and utility in a memory clinic setting. Canadian Journal of Psychiatry 2007; 52: 329-32. 
22. Luo XG, Feng Y, Liu R, et al. Cognitive deterioration rates in patients with Parkinson's disease from northeastern China. Dementia and Geriatric Cognitive Disorders 2010; 30: 64-70.

23. Fujiwara Y, Suzuki H, Yasunaga M, et al. Brief screening tool for mild cognitive impairment in older Japanese: validation of the Japanese version of the Montreal Cognitive Assessment. Geriatrics and Gerontology International 2101; 10: 225-32.

24. Sumathipala AMJ. New approach to translating instruments for cross-cultural research: a combined qualitative and quantitative approach for translation and consensus generation. International Journal of Methods in Psychiatric Research 2000; 9: 87-95.

25. Jones SR, Carley S, Harrison M. An introduction to power and sample size estimation. Emergency Medicine Journal 2003; 20: 453-8.

26. Luis CA, Keegan AP, Mullan M. Cross validation of the Montreal Cognitive Assessment in community dwelling older adults residing in the Southeastern US. International Journal of Geriatric Psychiatry 2009; 24: 197-201.

27. Coen RF, Cahill R, Lawlor BA. Things to watch out for when using the Montreal cognitive assessment (MoCA). International Journal of Geriatric Psychiatry 2011; 26: 107-8. 